RUNNING HEAD: Tactile elevation perception

\title{
TACTILE ELEVATION PERCEPTION IN BLIND AND SIGHTED PARTICIPANTS AND ITS IMPLICATIONS FOR TACTILE MAP CREATION
}

Sandra Jehoel, Paul T. Sowden, Simon Ungar, and Annette Sterr

Department of Psychology, University of Surrey, Guildford, GU2 7XH, UK

Address correspondence to Paul Sowden, Department of Psychology, University of Surrey, Guild ford, GU2 7XH, UK; p.sowden@ surrey.ac.uk

Please cite this article as:

Jehoel, S., Sowden, P.T., Ungar, S. and Sterr, A. (2009) Tactile elevation perception in blind and sighted participants and its implications for tactile map creation. Human Factors, 51, 208-223. 


\section{ABSTRACT}

Objective: To determine the optimal elevation of tactile map symbols. Background: Tactile perception research predicts that symbol elevation (vertical height) and texture on tactile maps could influence their readability. However, while research has shown that elevation influences detection and discrimination thresholds for single tactile stimuli, and that the physiological response of fingertip receptors varies with texture, little is known about the influence of these parameters on the identification of stimuli in the context of multiple symbols as found on tactile maps. Method: Sighted and visually impaired participants performed tactile symbol identification tasks. In Experiment 1, we measured the effect of elevation on identification accuracy. In Experiment 2, we measured the effect of elevation and symbol texture on identification speed. Results: Symbol elevation influenced both speed and accuracy of identification with thresholds being higher than found in work on detection and discrimination but lower than on existing tactile maps. Further, as predicted from existing knowledge of tactile perception, rough features were identified more quickly than smooth ones. Finally, visually impaired participants performed better than sighted ones. Conclusion: The symbol elevations necessary for identification $(0.040$ to $0.080 \mathrm{~mm})$ are considerably lower than would be expected on the basis of existing tactile maps (generally $0.5 \mathrm{~mm}$ or higher) and design guidelines $(0.4 \mathrm{~mm})$. Application: Tactile map production costs could be reduced and map durability increased by reducing symbol elevation. Further, legibility of maps could be improved by using rough features, which are read more easily, and smaller symbols, which reduces crowding of graphics.

\section{Keywords}

Symbol elevation, Tactile map, Haptic perception, Visual impairment 


\section{INTRODUCTION}

Tactile maps and diagrams are raised line pictures that are used to convey spatial information to people with visual impairments. This information can be of great importance to visually impaired people, as it allows them to study, work and live more independently. Tactile features, such as the raised lines on tactile maps, need to be produced at a sufficient size (which hereafter we use to refer to horizontal dimensions/2D area), and elevation (relief, vertical height) relative to a background surface, to be readable. Here, we discuss two studies that explore the effects of variation in tactile symbol elevation on their identification. We aimed to find the minimum elevation and size at which symbols could be identified accurately (study 1), and the minimum elevation at which tactile symbols could be identified quickly (study 2). We used experimental methods and results from psychophysical and neurophysiological research to inform the design of our studies, but aimed for greater ecological validity.

Currently, the elevation of tactile map features varies widely with, often, poor precision. Usually, the elevation of tactile symbols cannot be controlled accurately or is dictated by the production method, rather than being based upon knowledge of tactile perception. For example, thermoformed plastic does not impose an upper elevation limit and, as a consequence, images are often rather high; commonly well over $1 \mathrm{~mm}$ (Gardiner \& Perkins, 2002; undated). Features on swell paper are generally raised by around $0.5 \mathrm{~mm}$ and Braille embossers produce dots at elevations of 0.25 to $1.0 \mathrm{~mm}$ (Gill \& Silver, 2005). The printer developed in the Tactile Inkjet Mapping Project (TIMP) (McCallum \& Ungar, 2003a; 2003b), however, allows more accurate control over the elevation of the printed features by depositing multiple layers of polymer ink. This printer can produce tactile images at almost any elevation, in increments of $10 \mu \mathrm{m}$ on average, depending on type of ink, substrate material and curing process. With this new technology, an important balance needs to be 
struck between the need to minimize machine resources including ink $(3 \mathrm{ml}$ required to print an $\mathrm{A} 3$ print sheet at $5 \%$ coverage with an elevation of $500 \mu \mathrm{m})$ and printing time (30 seconds to print and cure one $10 \mu \mathrm{m}$ layer of ink onto an A3 sheet) while maximizing user performance. Therefore, it is important to find the minimum elevation at which tactile maps are easily read and understood. However, there is a lack of empirical research on symbol elevation. One set of guidelines (Tactimages, 2000) specifies a minimum elevation of 0.4 $\mathrm{mm}$, without reference to supporting evidence. However, most guidelines for tactile map design (Edman, 1992; Gardiner \& Perkins, 2002; Levi \& Rolli, 1994) simply emphasize that features need to be of a sufficient elevation, without specifying a minimum or optimal elevation.

Psychophysical studies can give an indication of the sensitivity of the fingertip to raised features. Spatial acuity of the finger has been studied, using methods including two point limen tests, tests on gap detection and letter recognition (Johnson \& Phillips, 1981; Stevens \& Cruz, 1996) as well as studies on tactile gratings (Craig, 1999; Johnson \& Phillips, 1981; Nefs et al., 2001; van Boven \& Johnson, 1994). These experiments suggest that the tactile spatial resolution of the fingertip ranges between 0.87 and $2.36 \mathrm{~mm}$. In other words, they indicate that the fingertip can be used to interpret tactile features (gaps between bars, the difference between two points and a single point, the difference between a horizontal and a vertical grating etc.), at some pre-defined criterion level of performance, only when the elements of these features are separated by more than the minimum resolution distance. Spatial acuity of the fingertip provides information to map designers about the minimum size of tactile symbols. Few studies have investigated tactile sensitivity to the elevation (third dimension) of tactile features, even though elevation is a crucial parameter for touch. A study on detection thresholds showed that a single edge can be detected at an elevation of $0.85 \mu \mathrm{m}$ relative to a flat background (Johansson \& LaMotte, 1983). In a study on the amplitude of 
gratings, participants discriminated amplitude (elevation) differences as small as $2 \mu \mathrm{m}$ (Nefs et al., 2001). In addition to elevation, the shape of an edge is important for detecting the tactile feature. Physiological studies show that sharp edges produce higher neural activity in the fingertip receptors than gradually sloping or curved edges (LaMotte \& Srinivasan, 1987).

Unfortunately, the results of psychophysical studies cannot be translated directly into decisions on tactile map design. Features that can be detected and discriminated in isolation on a single intensive trial of a psychophysical study, may not be correctly identified or may even be missed altogether when presented in the context of a complex tactile map. In short, tactile maps may not be readable at an elevation of $0.85 \mu \mathrm{m}$, even though this elevation is above the detection threshold. At the same time, psychophysical studies show that the fingertip is very sensitive to elevation, which suggests that tactile maps may be produced at considerably lower elevations than generally assumed. Currently, there is no satisfactory link between psychophysical studies on elevation and the general recommendations of guidelines for the design of tactile maps. We attempted to bridge this gap in two studies on the perception of the elevation of tactile symbols, using more practical and realistic tasks. Optimum elevations and sizes for identification have not been studied in psychophysical and neurophysiological studies before. However, they are the key issue for tactile map design; detection of a feature is useless if you cannot identify it.

Mechanoreceptors in the fingertip form the basis of tactile perception (for an overview of the tactile functions of mechanoreceptors, see Johnson et al., 2000). Exploration of small tactile symbols mainly engages responses in SA1 (Slowly Adapting) and RA (Rapidly Adapting) receptors (Phillips, et al., 1990). Accordingly, the design of our studies was determined by three strands of neurophysiological research on these receptors. Firstly, an increase in elevation was expected to improve performance, because of an increase in mechanoreceptor responses. For example, Blake, Johnson and Hsiao (1997b) found that the 
response of SA1 and RA mechanoreceptors increased with higher elevations. However, the effect of increasing elevation was expected to level off: at a certain elevation, any further increase in elevation was not expected to lead to meaningful gains in performance. This point would determine the optimal elevation, at which resources (ink and printing time) are minimized and performance is maximized. Thus, one of the aims of these studies was to identify the optimal elevation for tactile maps.

Secondly, we sought to determine the effect on performance of sharpness of edges. According to neurophysiological research, the mechanoreceptors in the fingertip are highly activated by sharp edges (LaMotte \& Srinivasan, 1987; Srinivasan \& LaMotte, 1987). When pressed onto or stroked across the fingertip, sharp edges triggered a larger neurophysiological response than smooth edges. Capitalizing on this edge effect, we hypothesized that tactile features consisting of sharp edges would be more discernible than those with smooth edges and would hence be readable at lower elevations.

Thirdly, we presumed that symbol identification could be improved further by taking into account the adaptation characteristics of mechanoreceptors. Whereas SA1 afferents continue to respond, RA afferents adapt to continual stimulation and cease to respond. This suggests that tracing a solid line may not activate the RA mechanoreceptors in an optimal way. However, an array of dots continued to activate both the SA1 and RA receptors when stroked across the fingertip (Blake et al., 1997a; Phillips et al., 1992). Therefore, we hypothesized that a dotted line would trigger responses in both receptor types and would, therefore, be easier to identify at low elevations than a smooth, continuous line.

Based on these considerations, we conducted two experiments, with sighted and visually impaired participants, to systematically address the identification of tactile symbols at various elevations and sizes. The first study investigated the minimum size and elevation at which individual tactile symbols can be identified accurately. Participants identified shapes at 
several elevations and sizes. Performance was measured by percentage correct identification. In the second study, we determined the elevation at which tactile features can be identified quickly in the context of a task designed to be more analogous to map reading. Participants performed a scanning task on arrays of symbols at several elevations and completion times were recorded. In order to study the interaction between line type and elevation, we designed three lines (smooth, sharp and rough), intended to take advantage of our knowledge of tactile perception of elevation, sharp edges and dot arrays. A rounded, smooth line was expected to trigger a relatively weak response of the mechanoreceptors and, therefore, require higher elevations. A line with a sharp edge was expected to trigger a stronger response and therefore require lower elevations. A rough line, consisting of dots, was expected to cause continual stimulation of both SA and RA receptors in the fingertip. Therefore, we expected the rough line to be readable at the lowest elevations.

Both visually impaired and sighted participants took part in these studies. Since tactile maps are used by visually impaired people, it was important to test elevation and size of tactile symbols with this user group. We were also interested in the performance of sighted participants without extensive experience with tactile symbols, as previous research suggests that visually impaired participants perform some tactile tasks faster and more accurately than sighted participants (Heller, 1989a; van Boven et al., 2000). If differences between visually impaired and sighted participants are found then the performance of the sighted participants could provide a baseline for the production of tactile maps for users with a recent visual impairment. 


\section{STUDY 1: ACCURACY OF SYMBOL IDENTIFICATION}

This study aimed to determine the lowest elevation at which individual symbols of different sizes could be accurately identified. We hypothesized that symbols would be more easily identified at higher elevations and at larger sizes.

\section{Method}

Participants. Nineteen volunteers took part in this study. Ten participants were sighted (6 male and 4 female, 19 to 30 years, mean age 22.3) and nine were visually impaired (5 male and 4 female, 18 to 62 years, mean age 29.1). Visually impaired participants were recruited through the Royal National College and the Surrey Association for Visual Impairment. Sighted participants were recruited at the University of Surrey and Anglia Ruskin University. Details of the visually impaired participants' characteristics can be found in Table 1.

Table 1 about here

Materials. Circles, squares and equilateral triangles were printed on matt PVC, blank business cards (54 x $85 \mathrm{~mm}$ ), using the TIMP printer (McCallum \& Ungar, 2003a; 2003b), in a range of sizes and elevations. The cards were made of rough plastic, as suggested by a previous study that explored map substrates (Jehoel et al., 2005a). Outline circles, squares and triangles were selected, because these are the most basic and most commonly used shapes for map symbols. One symbol was printed in the middle of each card. A pilot study was conducted to identify an appropriate range of sizes and elevations within which performance varied from chance to asymptote for each shape. Based on this, symbols were printed at six elevations $(7,14,22,30,38$ and $47 \mu \mathrm{m})$ and five sizes. The elevations of symbols listed here were verified by measuring the height of each symbol using a set of digital calipers. The diameters of the circles and the base lengths of the squares and triangles, and their corresponding 2D areas, are shown in Table 2. 
Table 2 about here

Except for the smallest triangle and largest square, all other shape/area combinations fell within the range of areas for at least one other shape making area an unreliable clue to symbol identity. The same is true of the symbol sizes except for the smallest square and largest triangles. In total, 90 cards were printed that accommodated all shapes, elevations and sizes.

The cards were presented in a wooden jig with slots for each card (see Figure 1). The slots were $14 \mathrm{~mm}$ apart on a horizontal line. Small indentations along the base of the jig indicated the position of the corresponding symbols. The jig contained three symbol cards, to be identified one at a time, together with a distracter card (see procedure) either side of the symbol cards. The jig was placed on a table in front of the participant and participants were asked to adjust the position of the jig so they were comfortable.

Figure 1 about here.

Procedure. Reading tactile maps often requires observers to retain information about recently scanned symbols in memory whilst identifying the currently felt symbol. Consequently, to maximize the ecological validity of the current task, we required participants to explore the first 'distracter' symbol in the jig and memorize it, then to identify each of the three symbols of interest here and finally to explore the second 'distracter' symbol in the jig and determine whether it matched the first. The distracter symbols were drawn from a large set of tactile map symbols (Jehoel et al., 2005b). The purpose of the distraction task was to impose a working memory load on participants whilst they carried out the symbol identification task such that conditions are likely to be more similar to the situation of identifying symbols on a real map. 
Participants were seated at a desk in a quiet room at their college or home. A forced choice paradigm was used. Participants were asked to identify each symbol as a circle, square or triangle. Sighted participants and those with residual vision were blindfolded. Every combination of size and elevation was presented 10 times to each participant. This resulted in 300 symbol presentations (6 elevations x 5 sizes x 10 presentations). For each particular symbol size and elevation combination a square, triangle or circle was randomly presented on a given trial and the overall order of the 300 symbol presentations was also randomized. Participants used one hand of their own choice, and were allowed to swap hands during the experiment. They scanned each card in turn, from left to right, and were not allowed to return to a previously scanned card. Scanning of each symbol was limited to five seconds. Imposing a time limit made sense in the context of map reading, because a map reader should not have to spend a large amount of time exploring a symbol. The experimenter observed participants' finger movements and started a timer as soon as the symbol was found. After five seconds, an auditory signal prompted participants to lift their finger off the symbols and to give a response. This procedure was repeated for each card.

\section{Results}

The total percentage of correct responses for each size-elevation combination was calculated, as an indication of accuracy of identification (see Figure 2). The data show that identification accuracy increased with size and elevation and that visually impaired participants identified the shapes more accurately.

Figure 2 about here.

To explore whether differences in identification accuracy between the shapes were statistically significant, a 3-way ANOVA (Visual Status (2) - sighted, visually impaired; Shape (3) - triangle, circle, square; Elevation (6) - 7, 14, 22, 30, 38 and $47 \mu \mathrm{m}$ ) with repeated measures on the latter two factors was conducted. For this analysis, because the levels of a 
size factor would differ by shape, scores were collapsed across the five sizes for each elevation. There was a main effect of shape $\left(F(1.52,25.78)=6.54, p<0.01\right.$, partial $\eta^{2}=0.28$, power $=0.88)$, but none of the interactions with shape were significant $(p>0.05)$. Bonferroni post hoc comparisons showed that triangles were identified significantly better than circles $(p<0.01)$, and neither differed from squares $(p>0.05)$ (Figure 2). Because the average area of the triangles was smaller than of the circles or squares (see Figure 2), and because identification improved with size, the better identification of the triangles cannot be attributed to the different range of sizes used across shapes.

Another way to illustrate this is to define some criterion level of performance and compare the size at which each shape can be identified against this criterion. If we consider the $80 \%$ correct level, which we define as our arbitrary criterion level of performance for the purposes of comparison, we can see that the lowest elevation that exceeds this criterion level for all shapes and both groups of participants is $22 \mu \mathrm{m}$. Figure 2 shows the point of intersection between this elevation and our $80 \%$ performance level for each shape and group. Reading off the $\mathrm{x}$-axis it can be seen that triangles are identifiable at smaller sizes than circles and squares. The better identification of the triangles may be because they have more acute angles making sharper points compared to the squares and circles. As noted earlier, physiological studies show that sharp edges produce higher neural activity in the fingertip receptors than gradually sloping or curved edges (LaMotte \& Srinivasan, 1987).

Having established the difference in performance between shapes, the effects of visual status, elevation and size on accuracy of symbol identification were explored using a separate ANOVA for each shape (see Table 3)

Table 3 about here.

There was a consistent pattern of main effects across the different shapes. However, there was no consistent pattern of interactions, despite moderate to good power for many of 
these, and the few interactions that were significant had small effect sizes, suggesting that there were no meaningful differences in the way that the two groups' performance varied at the various size/elevation combinations. Consequently, here we focus on summarizing the main effects.

First, participants with a visual impairment identified triangles and squares more accurately than sighted participants (Table 3 main effects of Visual Status; Figure 2a,b,e,f).

Second, identification performance increased with elevation at the lower elevations and then reached a shape dependent asymptote (Table 3 main effects of elevation; Figure 2) irrespective of size. Bonferroni post-hoc analysis showed that for triangles performance did not improve at elevations of $30 \mu \mathrm{m}$ and beyond, for circles this value was $14 \mu \mathrm{m}$ and for squares it was $22 \mu \mathrm{m}(p>0.05)$.

Third, identification performance increased with size before reaching a shape dependent asymptote (Table 3 main effects of size; Figure 2) irrespective of elevation. Bonferroni post-hoc analysis showed that for triangles performance did not improve at sizes of $17.49 \mathrm{~mm}^{2}$ or greater, for circles this value was $23.76 \mathrm{~mm}^{2}$ and for squares this value was $25 \mathrm{~mm}^{2}(p>0.0005)$; the middle size in each case (see Table 2).

\section{Discussion}

In this experiment, we studied the effect of symbol size and elevation on the accuracy of identification of tactile symbols. Participants identified simple shapes with five different sizes, at elevations ranging from 7 to $47 \mu \mathrm{m}$. As expected, both an increase in elevation and an increase in symbol size resulted in a higher accuracy of symbol identification. An elevation of $22 \mu \mathrm{m}$ was sufficient for reliable identification of individual symbols (defined here as $80 \%$ correct) in all cases.

The finding that increasing the area of the shapes beyond the middle value used for each shape did not further improve identification places an upper limit on the necessary size 
of, at least the present, tactile symbols on maps. This limit corresponds to base lengths of 6.4 $\mathrm{mm}$ for triangles, $5.0 \mathrm{~mm}$ for squares and a diameter of $5.5 \mathrm{~mm}$ for circles. Knowing these limits is useful because it is desirable to minimize crowding of symbols on maps by printing symbols at the smallest size at which identification performance reaches asymptote.

Finally, at ages of 51 and 62 two of the visually impaired participants were considerably older than all the other participants (mean age 21.82, range 18-33 years). Since tactile spatial acuity deteriorates with age at a rate of $1 \%$ threshold rise per year (Stevens et al., 1996), these two participants are likely to have reduced the mean identification performance of the visually impaired group relative to the sighted group. Despite this, the visually impaired group showed significantly better identification.

Although the results of this study suggest that tactile symbols are legible at very low elevations and small sizes, a further step is required to translate them directly into tactile map design. This is because in this study individual symbols were identified largely in isolation, whereas reading a tactile map is a more complex process. Consequently, in a second study we investigated the elevation of tactile symbols on symbol identification in a more realistic task.

\section{STUDY 2: SPEED OF SYMBOL IDENTIFICATION}

Study 1 yielded minimum elevations and sizes at which individual symbols can be identified accurately. This information provided a baseline for the second study, in which the elevation of tactile symbols was considered in a more realistic context. Given symbols that were large and high enough to be accurately identified, we explored the effects of further manipulating elevation and line type on the speed of identification of the symbols. We hypothesized that an increase in elevation would lead to faster performance, but that this improvement would level off at an optimal elevation. Further, we attempted to apply previous findings on the tactile perception of edges and dot patterns to create different line types that 
might make more effective stimuli. We hypothesized that rough and sharp lines would be easier to read than smooth lines.

\section{Method}

Participants. Nineteen volunteers took part in this study. Eight participants were sighted ( 2 male and 6 female, 18 to 30 years, mean age 22.5) and 11 were visually impaired (7 male and 4 female, 18 to 37 years, mean age 22.9). Visually impaired participants were recruited through the Royal National College and the Royal London Society for the Blind. Sighted participants were recruited at the University of Surrey. Details of the visually impaired participants' characteristics can be found in Table 4 .

Table 4 about here

Materials. Stimuli were printed on rough plastic, as suggested by the findings of Jehoel et al. (2005a), using the TIMP tactile printer. On the basis of crossing six elevations, three line types and two symbol arrays, 36 tactile displays were created. Symbols were printed at elevations of 20,40, 80, 160, 320 and $640 \mu \mathrm{m}$, using 'smooth', 'sharp' and 'rough' line types. The lowest elevation of $20 \mu \mathrm{m}$ was informed by the results of Study 1, as this was approximately the average elevation at which accuracy of symbol identification performance reached asymptote irrespective of symbol size. The smooth line was a solid line with a convex line profile, while the sharp line had a triangular profile. The rough lines consisted of two parallel rows of small dots. Figure 3 shows top views and cross sections of the line types. Due to limitations of the printing technique, rough lines were not printed at an elevation of $640 \mu \mathrm{m}$. Each tactile display consisted of a six by six array of outline circles and outline circles with gaps of $45^{\circ}$. Gaps were positioned at the top, bottom, left or right side of the circle. Seven full circles, acting as target symbols, were randomly located on each display. Displays could be rotated to produce four different versions of the symbol array. Circles were $2.5 \mathrm{~cm}$ in diameter and had a line width of $1.6 \mathrm{~mm}$. The circle arrays measured 20 by $20 \mathrm{~cm}$. 
To the left of each row, a horizontal line was printed that enabled participants to keep track of their position on the display. The arrays were placed on a table in front of the participant. Figure 4 shows an example of the displays.

Figure 3 about here.

Figure 4 about here.

Procedure. Participants were seated at a desk in a quiet room at their college or home. The experiment consisted of a tactile scanning task. Sighted participants and those with residual vision were blindfolded during the task. Participants were asked to scan the arrays of symbols as fast and as accurately as possible, proceeding from the top left corner to the bottom right corner and to give a verbal response when encountering a target symbol. Participants were asked to use their right hand to explore the rows of symbols, thereby ensuring that the visually impaired group could not use a different strategy involving use of both hands, learned from reading braille. All but one participant in each group was right handed. They used their left hand to keep track of their position by placing it on one of the horizontal lines to the left of each row. After a practice trial, 17 displays were presented in random order, representing all possible combinations of elevation and line type. A digital video camera was used to record the amount of time that was needed to complete the 6 by 6 arrays.

\section{Results}

The aim of this study was to investigate the effects of line type and elevation on performance of an identification task. We recorded the time it took participants to scan arrays of symbols, from the moment participants touched the first symbol in the display until they left the last symbol (see Figure 5).

Figure 5 about here 
The number of errors on each trial was very small $(M=.43, S D=1.1)$, including false positives and missed identifications of the target symbol. A Pearson correlation was used to determine the relationship between scanning time and number of errors. A negative correlation could indicate a trade off between speed and accuracy. However, the analysis showed that there was a positive correlation between scanning time and number of errors $(r=$ $.34, p<.001)$, and therefore there was no evidence of a trade off between speed and accuracy. This correlation suggests that participants' performance was both slower and less accurate on more difficult arrays.

The effects of visual status, elevation and line type on scanning time were analyzed using an ANOVA (see Table 5). Since rough lines were not printed at the highest elevation, this elevation was not included in the ANOVA.

Table 5 about here

The findings can be summarized as follows. First, visually impaired participants performed the task faster than sighted people (main effect of Visual Status; see Fig. 5a,b).

Second, scanning time decreased with increasing elevation (main effect of Elevation; see Fig. 5c). Bonferroni post hoc comparisons showed that there were significant differences in scanning time between all elevations $(p<0.01)$ that were included in the analysis except the difference between 80 and $160 \mu \mathrm{m}$ and the difference between 160 and $320 \mu \mathrm{m}$.

Third, scanning time varied significantly across line types (Fig. 5a,b,c). Bonferroni post hoc comparisons showed that rough lines were scanned faster than sharp and smooth lines $(p<0.05)$, and smooth lines were scanned faster than sharp ones $(p<0.05)$.

Fourth, visually impaired participants' search times were significantly faster at the two lowest elevations (interaction between elevation and visual status) of $20 \mu \mathrm{m}(\mathrm{p}<0.00005)$ and $40 \mu \mathrm{m}(\mathrm{p}<0.01)$ as assessed by Bonferroni post-hoc comparisons. 
Finally, performance tended towards asymptote sooner for the rough lines than for the sharp or smooth lines (interaction between line type and elevation; see Fig. 5c). Bonferroni post-hoc comparisons showed that for rough lines scanning time was significantly faster for $40 \mu \mathrm{m}$ than for $20 \mu \mathrm{m}$ elevations $(\mathrm{p}<0.001)$ but differences between the remaining, higher, adjacent elevations were not significant ( $\mathrm{p}>0.05$ ); for smooth and sharp lines the difference between $40 \mu \mathrm{m}$ and $80 \mu \mathrm{m}$ was also significant ( $\mathrm{p}<0.01$ and $\mathrm{p}<0.05$ respectively) and thereafter the differences between the remaining, higher, adjacent elevations were not significant $(\mathrm{p}>0.05)$. In addition, scanning time was significantly faster for rough lines than for smooth or sharp lines at an elevation of $40 \mu \mathrm{m}(\mathrm{p}<0.05$ and $\mathrm{p}<0.005$ respectively), but there were no significant differences $(\mathrm{p}>0.05)$ between any of the line types at elevations of $80 \mu \mathrm{m}$ or above.

In order to further explore the relationship between elevation and line type and, in particular, to draw conclusions with respect to the elevations to be used in tactile maps, we made power function fits (see Fig. 5c) to the average data from all participants in Study 2 . For comparison we also made separate fits to the data from the sighted and visually impaired participants (see Figure 5a,b). The fit parameters are shown in Table 6.

Table 6 about here

It can be seen that in all cases the $r^{2}$ values are high, indicating that the power functions describe the observed data well. The intercept parameters indicate the scanning time at an elevation of $1 \mu \mathrm{m}$. As expected from the preceding analysis, overall intercept values tend to be lower for the rough line types and higher for the sharp line types. The rate parameters indicate that overall increasing elevation leads to faster reductions in scanning time for the sharp lines than for the smooth lines and rough lines. Finally, we defined the point of diminishing returns for each function as the point at which a $1 \mu \mathrm{m}$ increase in elevation starts to yield less than a 1 second reduction in scanning time. This value could 
provide a useful guide to the optimal elevation to be used in tactile maps with the different line types. In general, these calculations indicate that lower elevations can be used with rough lines and that higher elevations are necessary for sharp lines. For instance, the value of $46 \mu \mathrm{m}$ for rough lines could be used as a guide to the required elevation of tactile symbols used on maps produced by the TIMP method and agrees well with the preceding post hoc analysis, which shows that scanning time does not decrease significantly beyond elevations of $40 \mu \mathrm{m}$ for these line types.

\section{Discussion}

In this study, participants performed a search task on an array of symbols at different elevations and line types and search times were recorded. Our findings confirm the hypothesis that an increase in elevation would lead to better performance, up to a certain elevation. Performance levels rose steeply by increasing the elevation of symbols up to 40 $\mu \mathrm{m}$ (rough) or $80 \mu \mathrm{m}$ (sharp/smooth), but a further increase in elevation did not affect performance.

Based on the findings of neurophysiological studies on mechanoreceptor responses to edges (LaMotte and Srinivasan, 1987; Srinivasan and LaMotte, 1987), we expected that sharp lines could be read at lower elevations than curved, smooth lines. This did not appear to be the case. On the contrary, we found that the sharp lines in our setting were more difficult to read at lower elevations. These results could be due to the shape of the line types. At similar elevations, triangular sharp lines contained a smaller amount of raised material than semicircular smooth lines. Possibly, the mechanoreceptors in the fingertip responded to the volume of the material, as well as to the elevation and edges. This volume effect did not appear in previous studies, since they investigated the mechanoreceptor response to a single edge on a stair-like step, which did not involve the same variation in volume as profiled lines. However, Goodwin et al. (1991) touched on this issue in their paper on the discrimination of 
curved surfaces. Circumventing the issue of volume differences, it might be possible to take advantage of the edge effect by using a rectangular line profile with two sharp edges and a similar volume to smooth lines.

We further found that rough lines were read more quickly than smooth and sharp lines. This was expected, as previous work suggests sharp edges, such as those of the dots, cause a stronger mechanoreceptor response and that dot patterns provide repeated presentation of edges as the finger traces the line, which may have caused a continual response in both RA and SA receptors (Phillips, Johansson et al., 1992). We also speculate that the orientation of 'attack' on the fingertip plays a role. Finger movement needs to be perpendicular to the orientation of a raised edge in order for the edge to be of maximum effect. When tracing a solid line, the edge of the line stays in more or less the same position on the fingertip and, therefore, may not cause maximum edge effect. The edge will be more perceptible if the finger scans at 90 degrees across the line, because the edge will move across a larger number of receptors. In fact, at the lowest elevations, some participants used a strategy of moving their finger across the lines to check for a gap in the circumference of each circle rather than tracing along the line, which anecdotally may be consistent with an intuitive attempt to increase the level of stimulation as described. In the case of a dotted line, the finger will always move perpendicularly across edges of individual dots, regardless of the orientation of movement of the finger.

This study also attempted to find the optimal elevation for tactile images, represented by the lowest elevation at which tactile symbols could be identified quickly such that further increases in elevation did not give rise to further meaningful increases in reading speed. At this elevation, resources (ink and printing time) are minimized and performance is maximized. Our approach to this was to identify the point of diminishing returns for each power function at which a $1 \mu \mathrm{m}$ increase in elevation led to less than a 1 second reduction in 
scanning time. In general the data indicate that, for all line types, the point of diminishing returns is reached at lower elevations for the visually impaired participants. On average, across all participants, the points of diminishing returns were $46 \mu \mathrm{m}, 64 \mu \mathrm{m}$ and $52 \mu \mathrm{m}$ for rough, sharp and smooth lines respectively. These points could be used as a guide to the optimal elevations for different line types on tactile maps. However, there was a nonsignificant trend of improved performance beyond the point of diminishing returns up to around $160 \mu \mathrm{m}$ after which performance is essentially flat, suggesting that the speed of map reading may be further facilitated by increasing elevation beyond the point of diminishing returns. Map designers must consider whether these further, non-statistically-significant, improvements in reading speed justify the increased time and expense of producing maps at these higher elevations.

\section{GENERAL DISCUSSION}

Our findings suggest a number of practical implications for tactile map production. First, our findings suggest suitable elevations for tactile map symbols. These elevations fall within the range between $40 \mu \mathrm{m}$ and $80 \mu \mathrm{m}$ depending on line type. Further, where production costs and time are not an issue performance gains may be possible beyond the point of diminishing returns up to $160 \mu \mathrm{m}$. These elevations are greater than the psychophysical threshold for tactile detection of $0.85 \mu \mathrm{m}$ measured in previous research (Johansson and LaMotte, 1983). However, our tasks required participants to identify symbols, as would be the case when reading a tactile map, and it is well known that identification thresholds are usually higher than detection and discrimination thresholds because identification requires increased processing. For instance, identification requires comparison of the felt stimulus to stored neural representations of the range of possible stimuli to be felt and selection of the best match. On the other hand these elevations are considerably lower than those used on current tactile maps $(0.25 \mathrm{~mm}$ to $>1 \mathrm{~mm}$ depending 
on type of map; see Introduction) or recommended by design guidelines (for example, Tactimages (2000) recommended a minimum elevation of $0.4 \mathrm{~mm}$ ) . This is because, to date, there has been little empirical research to identify appropriate symbol elevations and instead these have been largely determined by the limitations of existing production methods, which do not have the control over elevation that is possible with the TIMP process. Producing maps at lower elevations could have a number of benefits. In a deposition process like the TIMP printer, lower elevations can reduce printing time and the amount of ink being used, consequently reducing production costs. In addition, the use of lower elevations would be beneficial for production methods such as thermoform and multi-textural processes because they could facilitate storage, due to the thinner pages, and could create less vulnerable maps.

Second, the current studies suggest that rough lines can be read at lower elevations (46 $\mu \mathrm{m})$ than smooth $(52 \mu \mathrm{m})$ or sharp $(64 \mu \mathrm{m})$ lines. Maps produced by Braille embosser or microcapsule techniques, which automatically produce textured tactile features, already benefit from this effect. Map producers who use tactile inkjet, thermoform, or multi-textural processes can take advantage of this effect by creating rough or textured tactile features.

Third, decreasing symbol size could improve legibility and production of tactile graphics. Larger symbols take up more space on a map, resulting in excessively large or 'crowded' graphics. Smaller symbols would allow for smaller and/or less cluttered maps. Although the results of Study 1 should not be translated directly into map design, due to the limited ecological validity of the task, our findings suggest that symbols may be smaller than usually recommended (for example, APH (1997) and Tactimages (2000) suggest a size of $12.7 \mathrm{~mm}$ and $7 \mathrm{~mm}$ respectively compared to $<7 \mathrm{~mm}$ for all symbols tested here), and that the readability of small symbols may be improved by using higher elevations. It is interesting to note that our symbols required larger sizes (asymptotic performance at base lengths of 6.4 $\mathrm{mm}$ for triangles, $5.0 \mathrm{~mm}$ for squares and a diameter of $5.5 \mathrm{~mm}$ for circles) than the 
psychophysical spatial resolution research might suggest (estimates of spatial tactile resolution range between 0.87 and 2.36 mm (Craig, 1999; Johnson and Phillips, 1981; Nefs, et al., 2001; Stevens and Cruz, 1996; van Boven and Johnson, 1994)) and, as with elevation, we attribute this to the requirement for participants to identify stimuli.

In addition to considering the implications of these studies for the design of tactile maps, it is important to take into account user preference and experience. For example, although performance on the rough lines was better than on the two smooth line types, extended exposure to rough line types during map-reading might result in the fingertips becoming desensitized, or even painful. Further, regardless of the optimum elevation found in this study, users may happen to prefer higher lines. A previous study on the use of substrate materials (Jehoel et al., 2005a) showed that some users reported a preference for smoother background substrates while performing better on rougher substrates, indicating that preference may not simply be determined by effectiveness of use.

In both of the present studies, visually impaired participants performed better than sighted participants. This finding reinforces previous studies, which have shown that visually impaired participants perform better than sighted ones on certain tactile tasks (Craig, 1988; Easton \& Bentzen, 1980; Grant et al., 2000; van Boven et al., 2000), but not on others (Grant et al., 2000; Heller, 1988, 1989b; Pascual-Leone \& Torres, 1993). The differences are often explained by the relative contributions of tactile experience and the use of visual imagery in more complex tasks. The present study is not set-up to distinguish these possibilities. A further issue is that the performance disadvantage of the (blind-folded) sighted participants (and by implication the likely performance disadvantage of participants with a recent visual impairment) might rapidly reduce with practice. This would render the between group performance differences observed here less of a concern for map design as it could be expected that with a little practice the performance levels of the visually impaired group 
could be rapidly attained. On the other hand, if learning is slow to asymptote then map designers need to design maps with elevations that facilitate easy symbol identification even by participants with recent visual impairments. Thus, there is a clear need for further work to determine the rate at which learning asymptotes in these tasks.

Finally, in relation to the methods used, we believe that studies like the present ones can help to explore the relationship between more abstract psychophysical tasks and more concrete, ecologically valid tasks (see Jehoel et al. (2006) for a more detailed discussion of this approach). In the past it has been generally recognized that psychophysical findings cannot be directly applied to design (e.g. the two-point touch threshold is clearly smaller than the desirable separation of lines on a tactile map). However, the present study demonstrates the value of using knowledge gained from psychophysical and neurophysiological research in an applied context. Further studies remain to be carried out to determine the validity of the findings in this study to actual map contexts.

\section{ACKNOWLEDGEMENTS}

The research reported here was carried out with the support of a grant from the Engineering and Physical Science Research Council of the UK (EPSRC). The authors gratefully acknowledge the support of the other members of the Tactile Mapping Research Project (TIMP). We are also grateful to our participants who gave their time and attention to the research.

\section{REFERENCES}

American Printing House. (1997). Guidelines for design of tactile graphics. 2008, from http://www.aph.org/edresearch/guides.htm

Blake, D. T., Hsiao, S. S., \& Johnson, K. O. (1997a). Neural coding mechanisms in tactile pattern perception: The relative contributions of slowly and rapidly adapting 
mechanoreceptors to perceived roughness. The Journal of Neuroscience, 17(19), 7480-7489.

Blake, D. T., Johnson, K. O., \& Hsiao, S. S. (1997b). Monkey cutaneous SA1 and RA responses to raised and depressed scanned patterns: Effects of width, height, orientation, and a raised surround. Journal of neurophysiology, 78(5), 2503-2517.

Craig, J. C. (1988). The role of experience on tactual pattern perception: A preliminary report. International Journal of Rehabilitation Research, 11, 167-183.

Craig, J. C. (1999). Grating orientation as a measure of tactile spatial acuity. Somatosensory and Motor Research, 16(3), 197-206.

Easton, R. D., \& Bentzen, B. L. (1980). Perception of tactile route configuration by blind and sighted observers. Visual impairment and blindness, September, 254-265.

Edman, P. K. (1992). Tactile graphics. New York: American Foundation for the Blind.

Gardiner, A., \& Perkins, C. (2002). Best practise guidelines for the design, production and presentation of vacuum formed tactile maps. Retrieved 20/07/2005, from http://www.sed.manchester.ac.uk/geography/research/tactileguidelines/index.htm

Gardiner, A., \& Perkins, C. (undated). Best practise guidelines for the design, production and presentation of vacuum formed tactile maps. Retrieved 20/07/2005, from http://www.sed.manchester.ac.uk/geography/research/tactileguidelines/ind ex.htm

Gill, J., \& Silver, J. (2005). Braille cell dimensions. Retrieved 19/04/05, from www.tiresias.org

Goodwin, A., John, K. and Marceglia, A. (1991). "Tactile discrimination of curvature by humans using only cutaneous information from the fingerpads." Experimental Brain Research 86(3): 663-672. 
Grant, A. C., Thiagarajah, M. C., \& Sathian, K. (2000). Tactile perception in blind Braille readers: A psychophysical study of acuity and hyperacuity using gratings and dot patterns. Perception and Psychophysics, 62(2), 301-312.

Heller, M. A. (1988). Texture perception in sighted and blind observers. Perception and Psychophysics, 45(1), 49-54.

Heller, M. A. (1989a). Picture and pattern perception in the sighted and the blind: The advantage of the late blind. Perception, 18, 379-389.

Heller, M. A. (1989b). Tactile memory in sighted and blind observers: The influence of orientation and rate of presentation. Perception, 18, 121-133.

Jehoel, S., McCallum, D., Rowell, J., \& Ungar, S. (2006). An empirical approach on the design of tactile maps and diagrams: The cognitive tactualization approach. The British Journal of Visual Impairment, 24(2), 67-75.

Jehoel, S., Ungar, S., McCallum, D., \& Rowell, J. (2005a). An evaluation of substrates for tactile maps and diagrams: Scanning speed and users' preferences. Journal of visual impairment and blindness, 99(2), 85-95.

Jehoel, S., Ungar, S., McCallum, D., \& Rowell, J. (2005b). Discriminability of map symbols in tactile cartography. Paper presented at the Annual meeting of the American Association of Geographers, Denver, USA.

Johansson, R. S., \& LaMotte, R. H. (1983). Tactile detection thresholds for a single asperity on an otherwise smooth surface. Somatosensory Research, 1(1), 21-31.

Johnson, K. O., \& Phillips, J. R. (1981). Tactile spatial resolution i: Two-point discrimination, gap detection, grating resolution and letter recognition. Journal of Neurophysiology, 46(6), 1177-1191. 
Johnson, K. O., Yoshioka, T., \& Vega-Bermudez, F. (2000). Tactile functions of mechanoreceptive afferents innervating the hand. Journal of Clinical Neurophysiology, 17(6), 539-558.

LaMotte, R. H., \& Srinivasan, M. A. (1987). Tactile discrimination of shape: Responses of slowly adapting mechanoreceptive afferents to a step stroked across the monkey fingerpad. The Journal of Neuroscience, 7(6), 1655-1671.

Levi, F., \& Rolli, R. (1994). Manual of tactile graphics. Torino, Italy: Zamorani

McCallum, D., \& Ungar, S. (2003a). An introduction to the use of inkjet for tactile diagram production. The British Journal of Visual Impairment, 21(2), 73-77.

McCallum, D., \& Ungar, S. (2003b). Producing tactile maps using new inkjet technology: An introduction. Cartographic Journal, 40(3), 294-298.

Nefs, H. T., Kappers, A. M. L., \& Koenderink, J. J. (2001). Amplitude and spatial-period discrimination in sinusoidal gratings by dynamic touch. Perception, 30, 1263-1274.

Pascual-Leone, A., \& Torres, F. (1993). Plasticity of the sensorimotor cortex representation of the reading finger in braille readers. Brain, 116, 39-52.

Phillips, J. R., Johansson, R. S., \& Johnson, K. O. (1990). Representation of braille characters in human nerve fibers. Experimental Brain Research, 81, 589-592.

Phillips, J. R., Johansson, R. S., \& Johnson, K. O. (1992). Responses of human mechanoreceptive afferents to embossed dot arrays scanned across fingerpad skin. The Journal of Neuroscience, 12(3), 827-839.

Srinivasan, M. A., \& LaMotte, R. H. (1987). Tactile discrimination of shape: Responses of slowly and rapidly adapting mechanoreceptive afferents to a step indented into the monkey fingerpad. The Journal of Neuroscience, 7(6), 1682-1697. 
Stevens, J. C., \& Cruz, L. A. (1996). Spatial acuity of touch: Ubiquitous decline with aging revealed by repeated threshold testing. Somatosensory and Motor Research, 13(1), 110.

Stevens, J. C., Foulke, E., \& Patterson, M. Q. (1996). Tactile acuity, aging and Braille reading in long-term blindness. Journal of Experimental Psychology: Applied, 2(2), 91-106.

Tactimages, \& Training. (2000). Images tactiles - acces a la culture - formation: INS HEA. van Boven, R. W., Hamilton, R. H., Kauffman, T., Keenan, J. P., \& Pascual-Leone, A. (2000). Tactile spatial resolution in blind braille readers. Neurology, 54(12), 22302236.

van Boven, R. W., \& Johnson, K. O. (1994). The limit of tactile spatial resolution in humans: Grating orientation discrimination at the lip, tongue and finger. Neurology, 44(12), 2361-2366. 
Sandra Jehoel was Research Fellow at the Department of Psychology, University of Surrey, UK. She obtained her PhD in Psychology from the University of Surrey, UK, in 2008.

Paul Sowden is Senior Lecturer at the Department of Psychology, University of Surrey, UK. He obtained his $\mathrm{PhD}$ in Psychology from the University of Surrey, UK, in 1995.

Simon Ungar was Lecturer at the Department of Psychology, University of Surrey, UK. He obtained his $\mathrm{PhD}$ in Psychology from the University of Sheffield, UK, in 1994.

Annette Sterr is Professor at the Department of Psychology, University of Surrey, UK. She obtained her PhD in Psychology from the University of Konstanz, Germany, in 1998. 
Table 1: Characteristics of the blind and visually impaired participants in Study 1 (all information obtained from participants' verbal reports).

\begin{tabular}{cccccc}
\hline Age & Sex & Visual Status & of Impairment & Etiology & Braille \\
\hline 18 & $\mathrm{~F}$ & totally blind & birth & retinopathy of prematurity & $\mathrm{y}$ \\
20 & $\mathrm{M}$ & totally blind & birth & micro anophtalmia & $\mathrm{y}$ \\
33 & $\mathrm{~F}$ & totally blind & 6 months & retinal blastoma & $\mathrm{y}$ \\
23 & $\mathrm{M}$ & totally blind & 3 years & Leber's amaurosis & $\mathrm{y}$ \\
18 & $\mathrm{M}$ & totally blind & 9 years & detached retina & $\mathrm{y}$ \\
51 & $\mathrm{M}$ & totally blind & 22 years & glaucoma & $\mathrm{y}$ \\
18 & $\mathrm{~F}$ & residual vision & birth & retinopathy of prematurity & $\mathrm{n}$ \\
62 & $\mathrm{~F}$ & residual vision & birth & cataracts, scars, nystagmus & $\mathrm{y}$ \\
18 & $\mathrm{M}$ & residual vision & 5 years & alstrom syndrome & $\mathrm{y}$ \\
\hline
\end{tabular}


Table 2: Sizes of the shapes in Study 1.

\begin{tabular}{ccc}
\hline $\begin{array}{c}\text { Circle diameter in } \mathbf{~ m m} \\
\left.\text { (and area in } \mathbf{~ m m}^{\mathbf{2}}\right)\end{array}$ & $\begin{array}{c}\text { Triangle base length in } \\
\mathbf{m m}\left(\mathbf{a n d} \text { area } \text { in } \mathbf{~ m m}^{\mathbf{2}}\right)\end{array}$ & $\begin{array}{c}\text { Square base length in } \mathbf{~ m m} \\
\text { (and area in } \mathbf{~ m m}^{\mathbf{2}} \text { ) }\end{array}$ \\
\hline $3.9(11.95)$ & $4.5(8.78)$ & $3.6(12.96)$ \\
$4.7(17.35)$ & $5.4(12.74)$ & $4.3(18.49)$ \\
$5.5(23.76)$ & $6.4(17.49)$ & $5.0(25.00)$ \\
$6.3(31.17)$ & $7.3(22.93)$ & $6.8(33.64)$ \\
$7.1(39.59)$ & $8.2(29.11)$ & $6.5(42.25)$ \\
\hline
\end{tabular}


Table 3. Study 1: Results of three-way ANOVA's to explore the effects of visual status, elevation and size on percent correct identification of each shape (Visual Status (2) - sighted, visually impaired; Elevation (6) - 7, 14, 22, 30, 38 and $47 \mu \mathrm{m}$; Size (5) - see Table 2) with repeated measures on the latter two factors. The Greenhouse-Geisser correction to the degrees of freedom is made when the assumption of sphericity is violated (assessed by Mauchley's w). For all shapes there are significant main effects of Visual Status, Elevation and Size. There are a few significant interactions but these have only small effect sizes and are not consistent across shapes.

\begin{tabular}{|c|c|c|c|c|c|}
\hline Effect & $F$ & $d f$ & $\bar{p}$ & Partial Eta ${ }^{2}$ & $\begin{array}{c}\text { Observed } \\
\text { power }\end{array}$ \\
\hline \multicolumn{6}{|l|}{ Triangles } \\
\hline Visual Status & 27.71 & 1,17 & $<0.0001$ & 0.62 & 1.0 \\
\hline Elevation & 21.60 & $2.57,43.64$ & $<0.0001$ & 0.56 & 1.0 \\
\hline Size & 24.98 & 4,68 & $<0.0001$ & 0.60 & 1.0 \\
\hline Visual Status $\mathrm{x}$ Elevation & 1.13 & 5,85 & $>0.05$ & 0.06 & 0.38 \\
\hline Elevation $\mathrm{x}$ Size & 0.93 & $7.64,129.91$ & $>0.05$ & 0.05 & 0.71 \\
\hline Visual Status $\mathrm{x}$ Size & 3.99 & 4,68 & $<0.01$ & 0.19 & 0.89 \\
\hline $\begin{array}{l}\text { Visual Status x Elevation } \\
\mathrm{x} \text { Size }\end{array}$ & 1.14 & 20,340 & $>0.05$ & 0.06 & 0.82 \\
\hline \multicolumn{6}{|l|}{ Circles } \\
\hline Visual Status & 3.19 & 1,17 & $>0.05$ & 0.16 & 0.39 \\
\hline Elevation & 19.44 & $2.38,40.48$ & $<0.0001$ & 0.53 & 1.0 \\
\hline Size & 22.40 & $1.82,30.96$ & $<0.0001$ & 0.56 & 1.0 \\
\hline Visual Status $\mathrm{x}$ Elevation & 1.04 & $2.38,40.48$ & $>0.05$ & 0.06 & 0.35 \\
\hline Elevation $\mathrm{x}$ Size & 1.46 & $8.48,144.21$ & $>0.05$ & 0.08 & 0.92 \\
\hline Visual Status $\mathrm{x}$ Size & 0.50 & $1.82,30.96$ & $>0.05$ & 0.03 & 0.16 \\
\hline $\begin{array}{l}\text { Visual Status x Elevation } \\
\mathrm{x} \text { Size }\end{array}$ & 0.73 & $8.48,144.21$ & $>0.05$ & 0.04 & 0.57 \\
\hline \multicolumn{6}{|l|}{ Squares } \\
\hline Visual Status & 25.61 & 1,17 & $<0.0001$ & .60 & 1.0 \\
\hline Elevation & 13.94 & 5,85 & $<0.0001$ & 0.45 & 1.0 \\
\hline Size & 33.68 & 4,68 & $<0.0001$ & 0.66 & 1.0 \\
\hline Visual Status $\mathrm{x}$ Elevation & 2.72 & 5,85 & $<0.05$ & .14 & 0.80 \\
\hline Elevation $\mathrm{x}$ Size & 1.73 & $8.69,147.67$ & $>0.05$ & 0.092 & 0.97 \\
\hline Visual Status $\mathrm{x}$ Size & 1.82 & 4,68 & $>0.05$ & 0.097 & 0.53 \\
\hline $\begin{array}{l}\text { Visual Status x Elevation } \\
\mathrm{x} \text { Size }\end{array}$ & 3.26 & 20,340 & $<0.0001$ & 0.16 & 1.0 \\
\hline
\end{tabular}


Table 4: Characteristics of the blind and visually impaired participants in Study 2 (all information obtained from participants' verbal reports).

\begin{tabular}{|c|c|c|c|c|c|}
\hline \multirow[b]{2}{*}{ Age } & \multicolumn{4}{|c|}{ Age at Onset } & \multirow{2}{*}{$\begin{array}{l}\text { Braille } \\
\text { reader? }\end{array}$} \\
\hline & Sex & Visual Status & of Impairment & Etiology & \\
\hline 18 & $F$ & totally blind & Birth & retinopathy of prematurity & $y$ \\
\hline 18 & $\mathrm{~F}$ & totally blind & Birth & Leber's amaurosis & $y$ \\
\hline 19 & M & totally blind & Birth & unknown & $\mathrm{y}$ \\
\hline 20 & M & totally blind & Birth & Norrie's disease & $y$ \\
\hline 37 & M & totally blind & Birth & cerebral aneurism & $y$ \\
\hline 33 & $\mathrm{~F}$ & totally blind & 6 months & retinal blastoma & $y$ \\
\hline 18 & M & totally blind & 9 years & detached retina & $y$ \\
\hline 18 & M & totally blind & 14 years & retinoblastoma & learning \\
\hline 18 & M & residual vision & 5 years & alstrom syndrome & $y$ \\
\hline 21 & M & residual vision & 5 years & unknown & $y$ \\
\hline 35 & $\mathrm{~F}$ & residual vision & 15 years & glaucoma & $y$ \\
\hline
\end{tabular}


Table 5. Study 2: Results of a three-way ANOVA to explore the effects of visual status, elevation and line type on scanning time (Visual Status (2) - visually impaired, sighted; Elevation (5) - 20, 40, 80, 160 and $320 \mu \mathrm{m}$; Line Type (3) - 'smooth', 'sharp' and 'rough') with repeated measures on the latter two factors. The Greenhouse-Geisser correction to the degrees of freedom is made when the assumption of sphericity is violated (assessed by Mauchley's w). There are significant main effects of Visual Status, Elevation and Line Type and interactions between Visual Status and Elevation and between Elevation and Line Type.

\begin{tabular}{llllcc}
\hline Effect & $\boldsymbol{F}$ & $\boldsymbol{d} \boldsymbol{f}$ & $\boldsymbol{p}$ & Partial Eta $^{2}$ & Observed power \\
\hline Visual Status & 16.49 & 1,17 & $<0.001$ & 0.49 & 0.97 \\
Elevation & 49.68 & $1.2,20.9$ & $<0.001$ & 0.75 & 1.00 \\
Line Type & 24.28 & 2,34 & $<0.001$ & 0.59 & 1.00 \\
Visual Status x Elevation & 8.46 & $1.2,20.9$ & $<0.01$ & 0.33 & 1.00 \\
Elevation x Line Type & 3.70 & $1.9,32.5$ & $<0.05$ & 0.18 & 0.98 \\
Visual Status x Line Type & 0.72 & 2,34 & $>0.05$ & 0.04 & 0.16 \\
Visual Status x Elevation & 2.05 & $1.9,32.5$ & $>0.05$ & 0.11 & 0.81 \\
x Line Type & & & & & \\
\hline
\end{tabular}


Table 6: $Y$ intercept, and rate parameters of power function $\left(y=a x^{b}\right)$ fits to scanning time data of Study 2 for rough, sharp and smooth symbol arrays. The goodness of fit $\left(\mathrm{r}^{2}\right)$ and point of diminishing returns for each function are also indicated.

\begin{tabular}{cccccc}
\hline & Line & & & & Point of Diminishing \\
Participants & Type & Y intercept & Rate & $\mathbf{r}^{2}$ & Returns \\
\hline Visually Impaired & Rough & 171.22 & -0.22 & 0.96 & $21 \mu \mathrm{m}$ \\
Visually Impaired & Sharp & 547.00 & -0.41 & 0.97 & $47 \mu \mathrm{m}$ \\
Visually Impaired & Smooth & 346.67 & -0.33 & 0.95 & $36 \mu \mathrm{m}$ \\
Sighted & Rough & 932.36 & -0.43 & 0.93 & $67 \mu \mathrm{m}$ \\
Sighted & Sharp & 1243.50 & -0.44 & 0.99 & $80 \mu \mathrm{m}$ \\
Sighted & Smooth & 845.19 & -0.38 & 0.97 & $67 \mu \mathrm{m}$ \\
All & Rough & 497.98 & -0.36 & 0.93 & $46 \mu \mathrm{m}$ \\
All & Sharp & 874.46 & -0.43 & 0.98 & $64 \mu \mathrm{m}$ \\
All & Smooth & 580.57 & -0.36 & 0.98 & $52 \mu \mathrm{m}$ \\
\hline
\end{tabular}




\section{Figure captions}

Figure 1: Example of a wooden jig containing five cards as used in Study 1. The first and last cards (from left to right) are distracters (see procedure of Study 1). The three centre cards are examples of the symbols to be identified. The semi-circles below each card show the indentations in the jig to indicate the position of each card to the observer.

Figure 2: Study 1 accuracy of identification by symbol size for each elevation, shape and group. (a, c, e) Visually impaired participants. (b, d, f) Sighted participants. The dashed lines indicate our $80 \%$ performance criterion. For legibility, errors bars indicating \pm 1 standard error of the mean are only shown for the lowest and highest elevations.

Figure 3: A) Top view of outline circles used in Study 2. B) Corresponding cross sectional profiles of smooth, sharp and rough line types from Study 2.

Figure 4: Example of a stimulus array used in Study 2.

Figure 5: Study 2 mean scanning time of rough, sharp and smooth line arrays as a function of symbol elevation. (a) Visually impaired participants. (b) Sighted participants. (c) All participants. Power function $\left(y=\mathrm{ax}^{\mathrm{b}}\right)$ fits are shown for each line type. Error bars show \pm 1 standard error of the mean. 
Figure 1

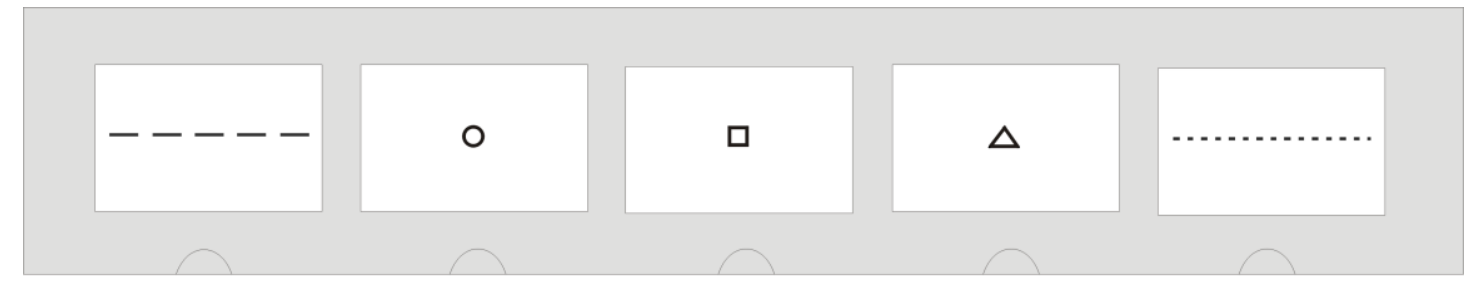


Figure 2
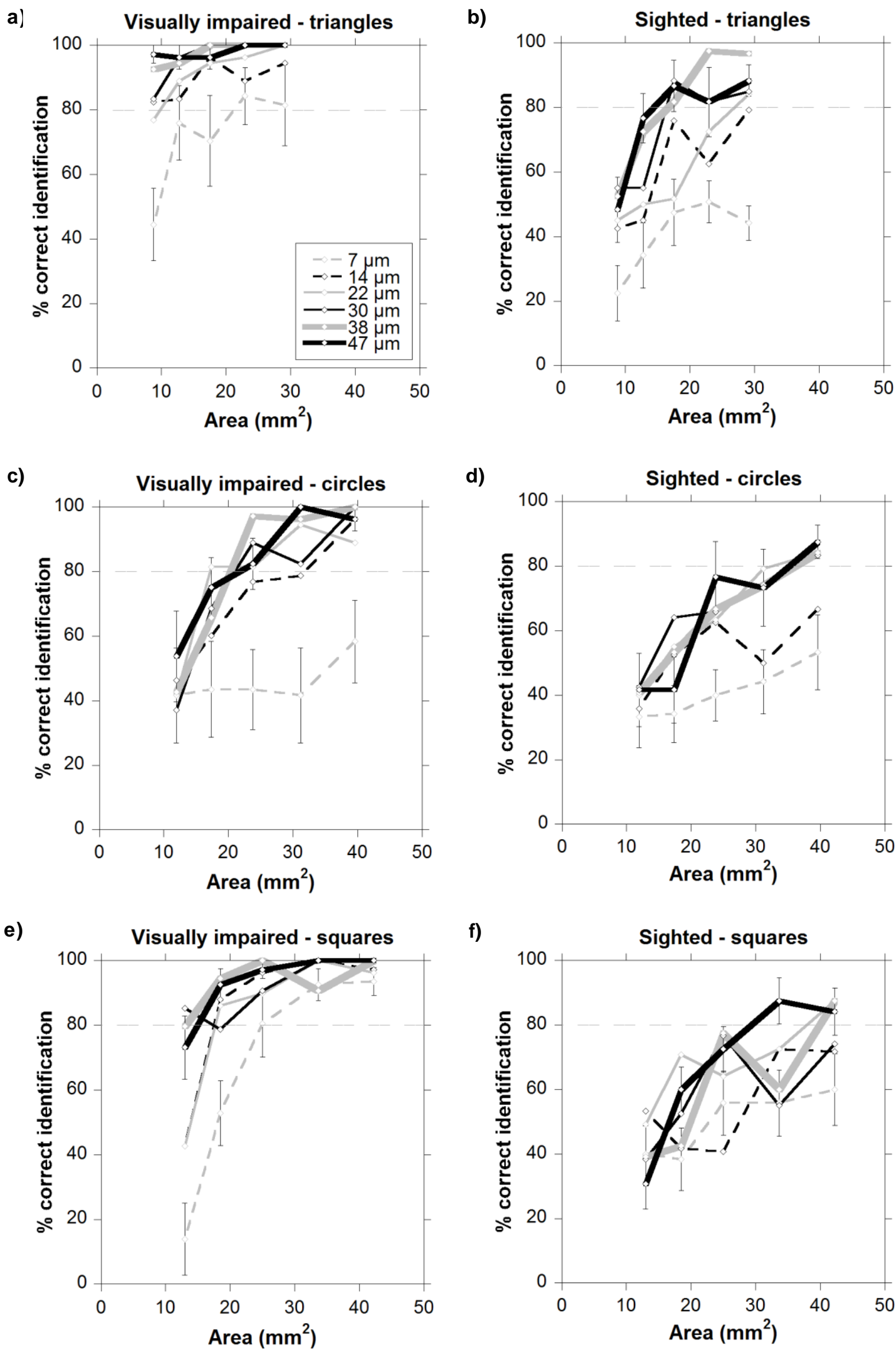
Figure 3

A

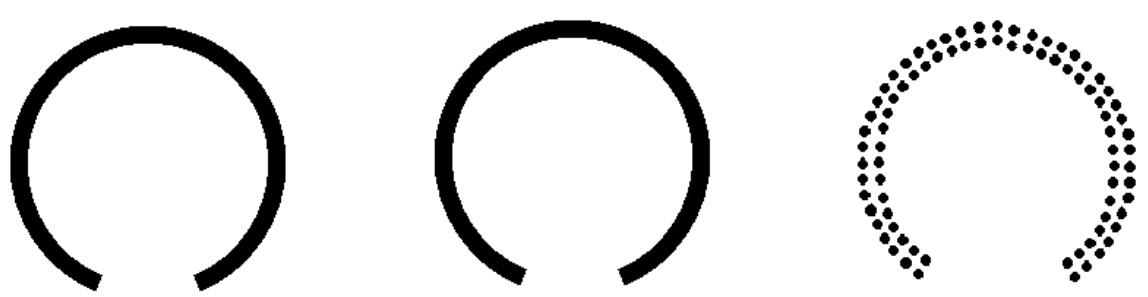

B

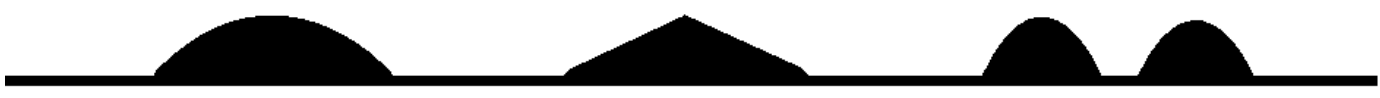


Figure 4

$$
\text { (1) }
$$


Figure 5

a)

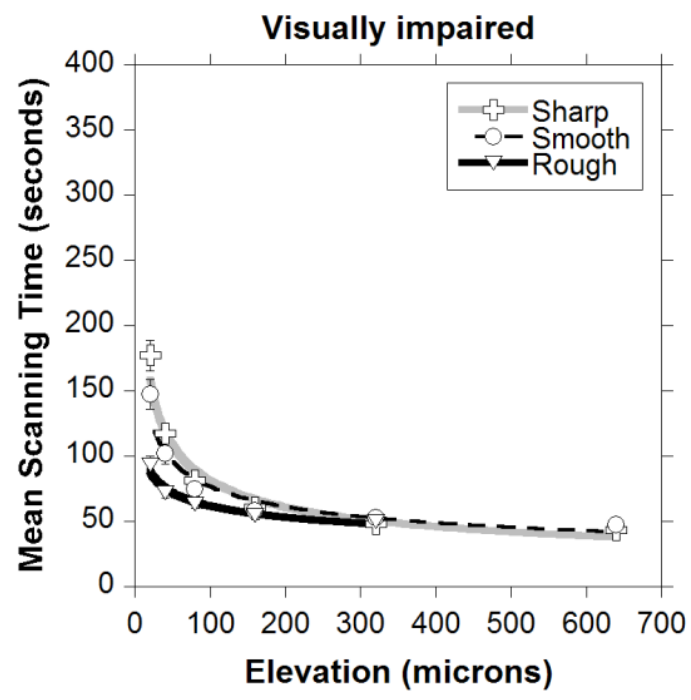

c)

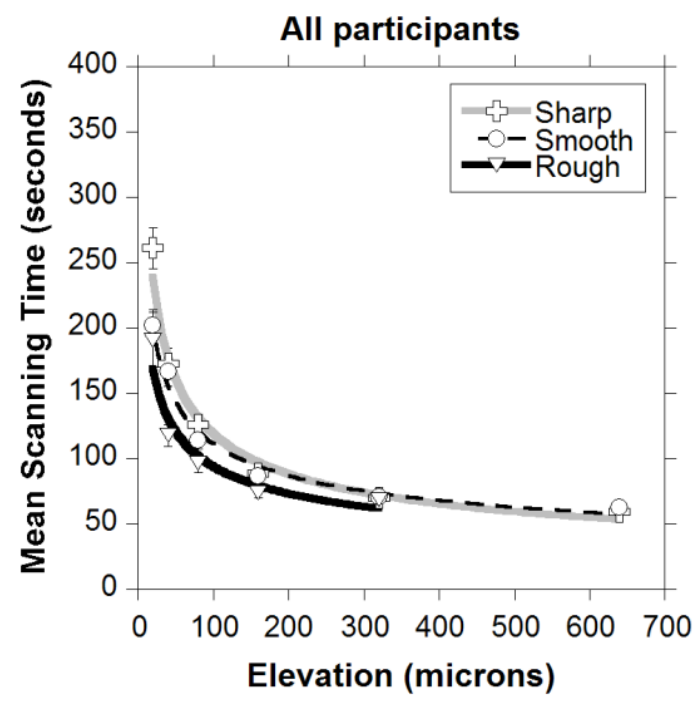

b)

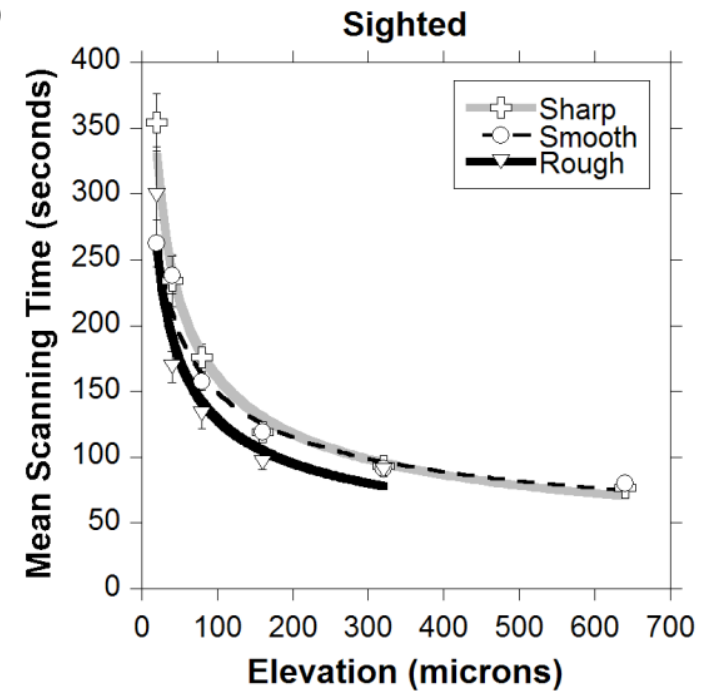

